\title{
Doping-dependent intraband carrier dynamics in Landau-quantized graphene
}

\author{
Florian Wendler* and Ermin Malic \\ Department of Physics, Chalmers University of Technology, SE-412 96 Göteborg, Sweden \\ (Received 6 October 2015; revised manuscript received 30 November 2015; published 19 January 2016)
}

\begin{abstract}
We investigate the intraband carrier dynamics in Landau-quantized graphene after an optical excitation with low-energetic terahertz pulses. Based on a microscopic theory, we calculate time-dependent differential transmission spectra reflecting the Landau-level dynamics. Our calculations reveal a strong dependence on the Fermi energy $E_{F}$ of the graphene sample as well as on the applied magnetic field $B$. We find that the pump pulse can lead to both absorption bleaching and absorption enhancement depending on $B$ and the position of $E_{F}$ with respect to the resonant Landau-level transition. As a result, positive and negative contributions in differential transmission spectra appear, in good agreement with recent pump-probe measurements.
\end{abstract}

DOI: 10.1103/PhysRevB.93.035432

\section{INTRODUCTION}

Applying a strong magnetic field perpendicular to a twodimensional electron gas causes a quantization of kinetic degrees of freedom and creates a discrete energy spectrum consisting of Landau levels (LLs) [1]. While for conventional two-dimensional materials with a parabolic electronic band structure the LL spacing is equidistant, graphene with its linear dispersion exhibits nonequidistant LLs with $\varepsilon_{\lambda n}=\lambda \hbar \omega_{c} \sqrt{n}$. Here, $\lambda= \pm 1$ denote the conduction and the valence bands, respectively, $\omega_{c}=\omega_{c}(B)$ stands for the cyclotron frequency, and $n$ describes the LL index. The optical selection rules [2-4] of Landau-quantized graphene $n \rightarrow n^{\prime}=n \pm 1$ allow intraband transitions $\left(\lambda=\lambda^{\prime}\right)$ between consecutive LLs as well as interband transitions $\left(\lambda \neq \lambda^{\prime}\right)$ with $\Delta n= \pm 1$. The nonequidistant Landau-level spectrum and the optical selection rules of Landau-quantized graphene allow the excitation of specific inter-LL transitions using circularly polarized light. As a result, one can track the path of excited electrons towards a thermalized Fermi distribution [5-16].

In this paper, we present a theoretical study of the intraband carrier dynamics in Landau-quantized graphene after excitation with low-energy terahertz pulses. The applied microscopic approach is based on the density matrix formalism, a wellestablished method for modeling the many-particle-induced carrier dynamics in graphene with and without the influence of a magnetic field [9,15,17-19]. In accordance with a recent pump-probe experiment [14], we consider terahertz photons with an energy of $14 \mathrm{meV}$ which is well below the cyclotron energy $\hbar \omega_{c} \simeq 36 \mathrm{meV} \sqrt{B}$ at magnetic field strengths of $B=1 \mathrm{~T}$. In conventional semiconductors with an equidistant Landau quantization, the absorption of low-energy terahertz photons is generally strongly suppressed, unless the cyclotron energy coincides with the photon energy. In contrast, one can always find an approximately resonant transition $\mathrm{LL}_{n} \rightarrow \mathrm{LL}_{n+1}$ matching the photon energy in graphene due to the nonequidistant Landau-level spacing (see Fig. 1). However, these transitions can only be induced by optical pumping if the involved levels $\mathrm{LL}_{n}$ and $\mathrm{LL}_{n+1}$ are not fully occupied or entirely empty. Hence, we expect the intraband carrier

\footnotetext{
*florian.wendler@chalmers.se
}

dynamics to be strongly dependent on the Fermi energy $E_{F}$ for a given magnetic field $B$.

Figure 1(a) illustrates that resonances between the fixed photon energy and inter-Landau-level transitions $\mathrm{LL}_{n} \rightarrow \mathrm{LL}_{n+1}$ occur at specific magnetic fields $B$ (see the yellow arrows), where the required $B$ increases with the LL index $n$. Furthermore, in order to resonantly absorb the incident photons, the Fermi energy has to be located in the gap between the involved $\mathrm{LL}_{n}$ and $\mathrm{LL}_{n+1}$ [for low temperatures and small LL broadenings; see Fig. 1(b)]. Interestingly, we observe a linear dependence between the required Fermi energies and magnetic fields at the resonant condition. Therefore, to obtain a considerable absorption of low-energy photons in graphene, the Fermi energy has to lie on a strip of constant slope when plotted against the magnetic field [see gray region in Fig. 1(a)]. To calculate the slope of

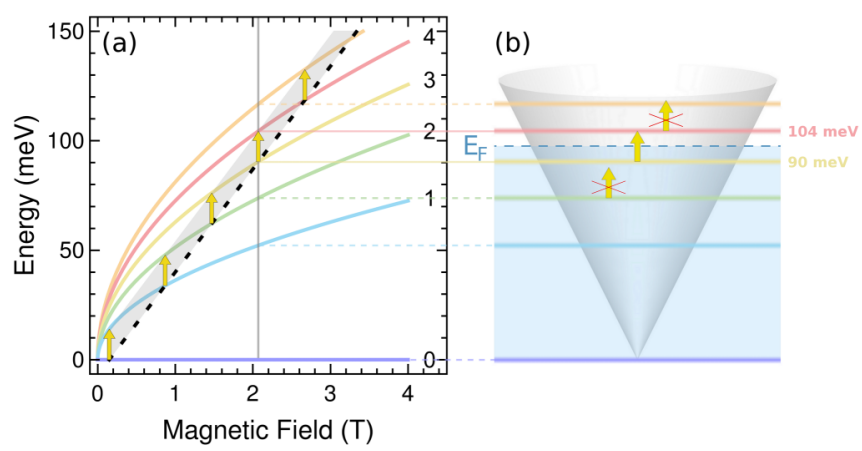

FIG. 1. Optically allowed intraband Landau-level transitions at a fixed terahertz excitation. (a) Energy of the energetically lowest LLs depending on the magnetic field $B$. At specific $B$, optically allowed inter-LL transitions are in resonance with the applied terahertz pulse with a fixed energy of $14 \mathrm{meV}$ (yellow arrows). Interestingly, the arrows lie on a straight line, as indicated by the gray region. The lower boundary of the gray region, the dashed black line, is given by Eq. (3). (b) Sketch of the Landau-level spectrum for a magnetic field of $B=2.1 \mathrm{~T}$ with the Dirac cone in the background. At this $B$ value, the inter-LL transition $\mathrm{LL}_{3} \rightarrow \mathrm{LL}_{4}$ is resonantly excited. This allows efficient absorption of low-energy photons, provided that the Fermi energy lies in the gap between $\mathrm{LL}_{3}$ and $\mathrm{LL}_{4}$. In this case, the transitions $\mathrm{LL}_{2} \rightarrow \mathrm{LL}_{3}$ and $\mathrm{LL}_{4} \rightarrow \mathrm{LL}_{5}$ are not only off-resonant but are also strongly suppressed due to Pauli blocking (see the crossed-out arrows). 
this strip, we start with the resonance condition

$$
\varepsilon_{\text {light }}=\varepsilon_{\lambda n+1}-\varepsilon_{\lambda n}=\hbar \omega_{c}(B)[\sqrt{n+1}-\sqrt{n}] .
$$

Solving for the Landau-level index $n$, we obtain a $B$-dependent expression,

$$
n(B)=\left(\frac{c^{2}-1}{2 c}\right)^{2}, \quad c=\frac{\hbar \omega_{c}(B)}{\varepsilon_{\text {light }}},
$$

that fulfills the resonance condition. Inserting this expression into Eq. (1) yields the corresponding Fermi energy

$$
E_{F}=\lambda\left(\frac{\hbar^{2} \omega_{c}^{2}(B)}{2 \varepsilon_{\text {light }}}-\frac{\varepsilon_{\text {light }}}{2}\right)=\lambda\left(\frac{\hbar e_{0} v_{F}^{2}}{\varepsilon_{\text {light }}} B-\frac{\varepsilon_{\text {light }}}{2}\right),
$$

with the cyclotron frequency $\omega_{c}(B)=\sqrt{\frac{2 e_{0} B}{\hbar}} v_{F}$, where $v_{F}=1 \mathrm{~nm} \mathrm{fs}^{-1}$ is the Fermi velocity [20]. As a result, it is crucial to investigate the intraband dynamics of optically excited charge carriers in Landau-quantized graphene as a function of the magnetic field $B$ and the Fermi energy $E_{F}$.

\section{THEORETICAL APPROACH}

\section{A. Semiconductor Bloch equations}

To microscopically model the Landau-level dynamics, we apply the Bloch equations of Landau-quantized graphene [15],

$$
\begin{gathered}
\dot{\rho}_{i}=-2 \sum_{l} \operatorname{Re}\left[\Omega_{i l} p_{i l}\right]+S_{i}^{\mathrm{in}}\left(1-\rho_{i}\right)-S_{i}^{\mathrm{out}} \rho_{i}, \\
\dot{p}_{i f}=\frac{i}{\hbar}\left(\epsilon_{f}-\epsilon_{i}\right) p_{i f}-\Omega_{i f}^{*}\left(\rho_{f}-\rho_{i}\right)-\gamma p_{i f},
\end{gathered}
$$

describing the temporal evolution of the carrier occupation $\rho_{i}$ in the state $i=\{\xi, \lambda n, m, s\}$ and of the microscopic polarization $p_{i f}$, which is a measure of the optical transition probability between states $i$ and $f$. The quantum numbers describing these states contain the valley index $\xi= \pm 1$, the band index $\lambda= \pm 1$, and the LL index $n=0,1,2, \ldots$, as well as the angular momentum $n-m=n, n-1, n-2, \ldots$ and the spin $s=$ $\pm 1 / 2$. To minimize the effect of an initial thermal excitation of the system, a low temperature of $T=10 \mathrm{~K}$ is assumed, corresponding to the value in the previous experimental investigation of the intraband carrier dynamics in Landau-quantized graphene [14]. The appearing Rabi frequency $\Omega_{i f}$ contains the optical excitation field as well as the optical matrix element, which determines the optical selection rules. The rates $S_{i}^{\text {in/out }}$ describe the phonon-induced scattering into and out of the $\mathrm{LL}_{i}$, respectively. Here, we neglect the impact of carrier-carrier interaction on the LL dynamics since the occupations of only a small number of Landau levels around the Fermi energy are changed by low-energy terahertz radiation. Consequently, the energy-conserving carrier-carrier scattering channels are strongly suppressed by Pauli blocking [9,21]. We have verified the validity of this approximation by performing calculations with and without taking into account the Coulomb interaction in different regimes. Since the optical phonon energies are much larger than the low-energy photons [15], the phononinduced relaxation should be also negligible [6]. However, to account for the rather fast experimentally observed decay of differential transmission [13,14], carrier-phonon scattering is included on a phenomenological level.
The dephasing $\gamma$ is assumed to be governed by the carrierimpurity scattering. It is explicitly calculated by exploiting the self-consistent Born approximation [9,15,22]. In good approximation, it results in a Landau-level broadening of $\hbar \gamma=v_{F} \sqrt{\hbar e_{0} B / A_{\text {imp }}}$ that depends on the Fermi velocity, the free-electron charge $e_{0}$, the magnetic field $B$, and the parameter $A_{\text {imp }}$ determining the carrier-impurity interaction strength [22]. We set $A_{\text {imp }}=165$ to obtain a broadening of $2 \mathrm{meV}$ at $B=1 \mathrm{~T}$, corresponding to the values in a recent pump-probe experiment [14]. According to the Fermi-Dirac distribution, the initial values of the occupations depend on the Fermi energy $E_{F}$. As described in Ref. [13], $E_{F}$ in Landau-quantized graphene is extracted from the Fermi energy in the absence of a magnetic field using the assumption that the carrier concentration does not change when a magnetic field is switched on.

\section{B. Differential transmission spectrum}

The derived graphene Bloch equations allow microscopic access to the time- and energy-resolved dynamics of optically excited carriers. Having the knowledge of the temporal evolution of the occupation probabilities, we can also calculate the differential transmission spectrum (DTS) that is directly accessible in pump-probe experiments. For a linearly polarized probe pulse of the frequency $\omega$ that is incident with a temporal delay of $\tau$ after the pump pulse (with the same energy and polarization), we obtain [23]

$$
\begin{aligned}
\operatorname{DTS}(\omega, \tau) \propto & \frac{1}{\omega} \sum_{\lambda_{i} n_{i}, \lambda_{f} n_{f}}\left(\alpha_{n_{i}} \alpha_{n_{f}}\right)^{2}\left(\delta_{n_{f}, n_{i}+1}+\delta_{n_{f}, n_{i}-1}\right) \\
& \times\left[\Delta \rho_{f}(\tau)-\Delta \rho_{i}(\tau)\right] \frac{\gamma_{i f}}{\Delta \omega_{i f}^{2}+\gamma_{i f}^{2}},
\end{aligned}
$$

where $\Delta \omega_{i f}=\omega-\left(\epsilon_{f}-\epsilon_{i}\right) / \hbar$ is the energy difference between the excitation pulse and the investigated inter-LL transition $i \rightarrow f$. Furthermore, $\Delta \rho_{j}(\tau)=\rho_{j}(\tau)-\rho_{j}^{0}$ is the occupation change induced by the pump pulse (compared to the initial thermal carrier distribution $\rho_{j}^{0}$ ). The appearing factor $\alpha_{n}$ stems from the tight-binding wave functions and reads $\alpha_{n=0}=\sqrt{2}$ for the zeroth LL and $\alpha_{n \neq 0}=1$ otherwise. Inspecting Eq. (6), we observe that the DTS is determined by the sum over pump-induced occupation changes in the final and initial states of all optically allowed transitions. Here, every transition is weighted by a Lorentzian centered around the resonant frequency $\omega=\left(\epsilon_{f}-\epsilon_{i}\right) / \hbar$.

We apply a strong pump pulse to excite the carrier system and calculate the differential transmission of a much weaker probe pulse with the same energy. If the system absorbs a fraction of the incoming radiation, the differential transmission is expected to be positive since pumping increases the Pauli blocking and leads to reduced absorption, i.e., increased transmission, of the probe pulse. This statement is based on the assumption that the same electronic transitions are pumped and probed. However, if different transitions are addressed with the pump and the probe pulse, the DTS can also be negative due to absorption enhancement. This can be achieved, e.g., by pumping and probing with light of different polarization [13] or by using a two-color pump-probe setup. 
(a)

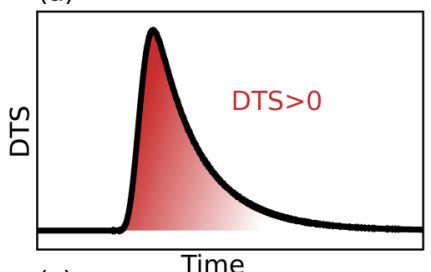

(c)

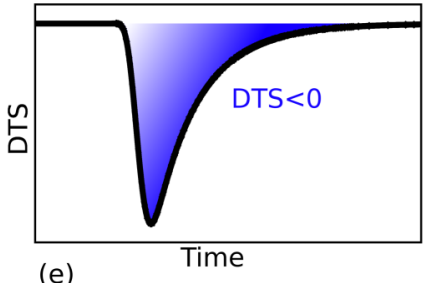

(e)

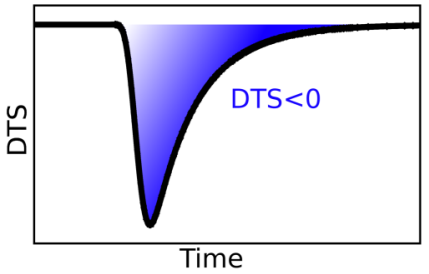

(b) PUMP PROBE

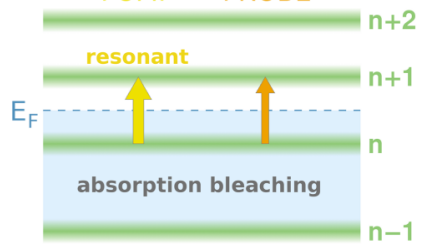

(d)

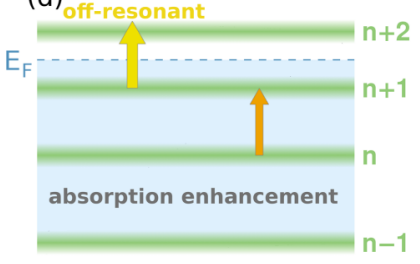

(f)

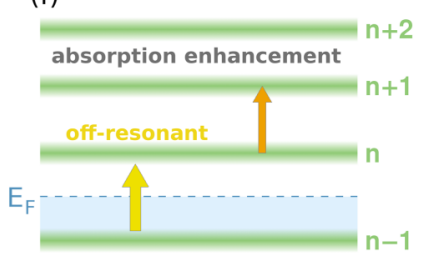

FIG. 2. Expected behavior in differential transmission spectra (DTS) for different Fermi energies $E_{F}$. (b) In the case of $E_{F}$ located between the two Landau levels of the resonant transition, we expect (a) a positive DTS due to absorption bleaching. If $E_{F}$ is located (d) above or (f) below, the pump pulse excites off-resonant transitions (yellow arrows), leading to an enhancement of the probe pulse absorption (orange arrows) and hence (c) and (e) a negative DTS.

Remarkably, applying low-energy terahertz radiation, a negative DTS can be obtained even if the pump and probe pulse have the same energy and polarization. This is due to the fact that absorption does not take place only for resonant pumping; there are also off-resonant contributions that can become crucial in certain situations. In Fig. 2, the expected DTS for three different positions of the Fermi energy $E_{F}$ with respect to the resonant LL transition $\mathrm{LL}_{n} \rightarrow \mathrm{LL}_{n+1}$ is shown. If $E_{F}$ is above the upper level of the resonant LL transition [see Figs. 2(c) and 2(d)], the transition cannot be pumped directly since the involved LLs are fully occupied and the strong Pauli blocking suppresses any transitions between these LLs. Instead, pumping induces the energetically closest off-resonant transition $\mathrm{LL}_{n+1} \rightarrow \mathrm{LL}_{n+2}$ [see the yellow arrow in Fig. 2(d)]. Thereby, the absorption of the probe pulse is enhanced since the occupation of the final state $L_{n+1}$ is reduced. In this situation, we expect a negative DTS. A similar behavior is expected if the Fermi energy is located below the resonant transition [see Figs. 2(e) and 2(f)]. Here, the initial state of the probe pulse absorption becomes occupied through the nonresonant pumping of the transition $\mathrm{LL}_{n-1} \rightarrow \mathrm{LL}_{n}$. This results in an enhanced absorption and a negative DTS. Only in the case where the Fermi energy lies in the gap of the resonant LL transition is the excitation from the pump pulse not blocked, and we expect an absorption bleaching resulting in a positive DTS [see Figs. 2(a) and 2(b)].

The importance of off-resonant contributions crucially depends on the magnitude of the energy mismatch and on the broadening of the Landau levels. As can be seen in

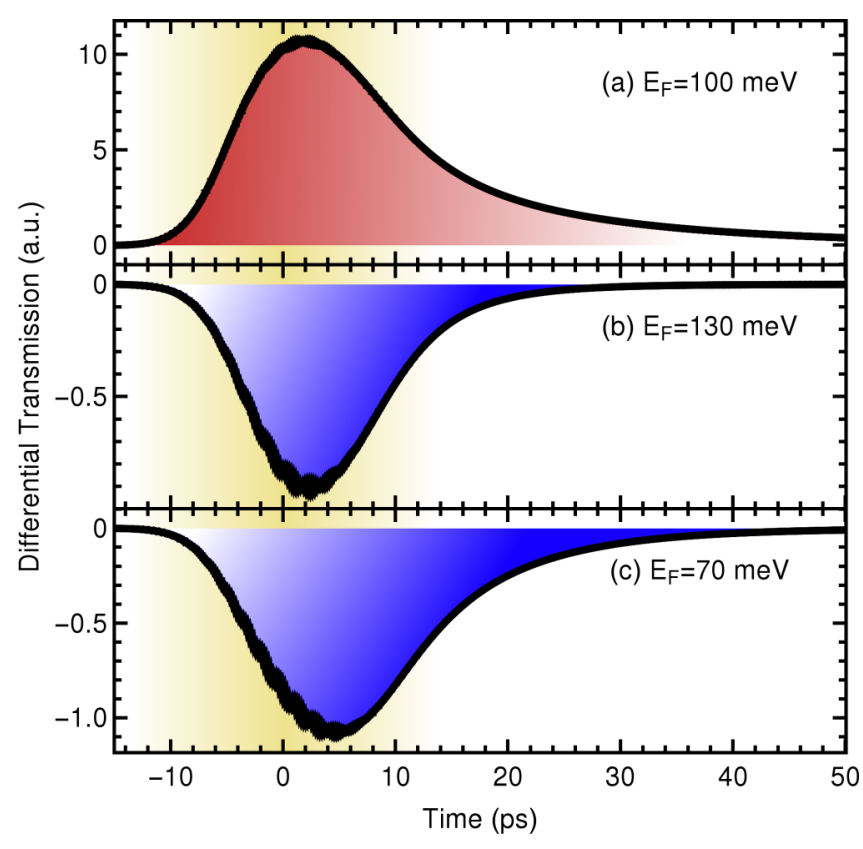

FIG. 3. Calculated temporal evolution of differential transmission. A linearly polarized excitation pulse with an energy of $14 \mathrm{meV}$, a pulse width of $10 \mathrm{ps}$ (yellow area in the background), and a pump fluence of $0.02 \mu \mathrm{J} \mathrm{cm} \mathrm{cm}^{-2}$ is applied. Furthermore, a magnetic field of $2.1 T$ is used, at which the transition $\mathrm{LL}_{3} \rightarrow \mathrm{LL}_{4}$ is resonantly excited (see Fig. 1). Here, we model the three representative cases presented in Fig. 2: (a) Fermi energy $E_{F}=100 \mathrm{meV}$ between $\mathrm{LL}_{3}$ and $\mathrm{LL}_{4}$, (b) $E_{F}=130 \mathrm{meV}$ well above $\mathrm{LL}_{4}$, and (c) $E_{F}=70 \mathrm{meV}$ well below $\mathrm{LL}_{3}$.

Fig. 1(a), the distance between neighboring LLs decreases with the Landau-level index $n$, and so does the energy mismatch between two consecutive transitions, $\mathrm{LL}_{n-1} \rightarrow \mathrm{LL}_{n}$ and $\mathrm{LL}_{n} \rightarrow \mathrm{LL}_{n+1}$. The impact of off-resonant pumping with low-energy radiation increases with the magnetic field since then transitions involving LLs with a higher index $n$ become resonantly excited [see Fig. 1(a)]. This is further amplified by the fact that also the impurity-induced Landau-level broadening increases with the magnetic field $[9,21,22]$.

\section{RESULTS}

\section{A. Temporal evolution of differential transmission}

Solving the coupled set of differential equations [Eqs. (4) and (5)] and calculating the differential transmission according to Eq. (6), we have access to the intraband Landau-level dynamics. Figure 3 shows the differential transmission at a constant magnetic field of $2.1 T$ for different Fermi energies $E_{F}$. The chosen magnetic field corresponds to the case sketched in Fig. 1(b), at which a resonance between the photon energy and the LL transition $\mathrm{LL}_{3} \rightarrow \mathrm{LL}_{4}$ is generated. The Fermi energy in Fig. 3 lies either between $\mathrm{LL}_{3}$ and $\mathrm{LL}_{4}$ or is located well above $\mathrm{LL}_{4}$ or well below $\mathrm{LL}_{3}$. In agreement with the expectation from Fig. 2, we observe a positive DTS for $E_{F}$ between the LLs of the resonant transition [Fig. 3(a)], while a negative DTS is obtained for Fermi energies above and below [Figs. 3(b) and 3(c)]. Furthermore, note that the absolute value of the DTS strongly differs: Since the pump pulse in the latter two cases can only induce off-resonant transitions due to 


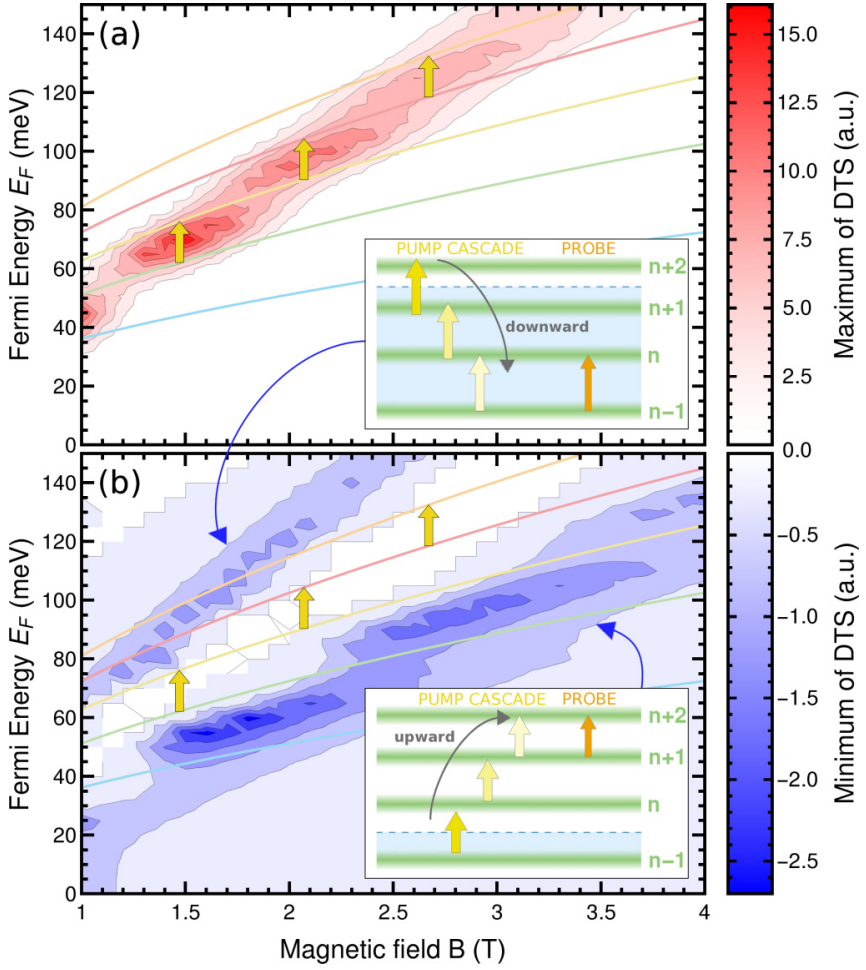

FIG. 4. Differential transmission as a function of Fermi energy $E_{F}$ and magnetic field $B$. Illustrations of appearing (a) maxima and (b) minima in the DTS depending on the position of $E_{F}$ and the strength of $B$. The lines represent the Landau-level energies, and the yellow arrows indicate resonances with the radiation field [see Fig. 1(a)]. The insets illustrate schematically the cascade pumping.

the entirely occupied LLs and the corresponding strong Pauli blocking, the amplitude of the DTS is significantly suppressed. Interestingly, in certain situations, the temporal evolution of the differential transmission can have both negative and positive parts (not shown).

\section{B. Dependence of DTS on magnetic field and Fermi energy}

The dependence of the DTS on the magnetic field and on the Fermi energy is further illustrated in a surface plot that exhibits clearly appearing maxima and minima in the DTS (see Fig. 4). The maxima correspond to cases where resonant absorption of low-energy radiation is possible. Here, the Fermi energy lies between the two LLs of the resonant transition [see Fig. 4(a)]. Figure 4(a) illustrates nicely the clear linear dependence between the magnetic field and the Fermi energy [see Eq. (3) and Fig. 1(a)]. The slope is $47 \mathrm{meV} / \mathrm{T}$, which agrees well with Eq. (3). Figure 4(b) shows the DTS minima corresponding to the negative contributions that are present if the Fermi energy lies above or below the resonant transition [yellow arrows; see Figs. 2(b) and 2(c), respectively]. The negative contributions of the DTS correspond to situations where off-resonant pumping leads to an induced absorption of the resonant probe pulse.

Interestingly, the minima are not located at the resonant magnetic fields, but they are shifted to higher or lower $B$. There is, e.g., a clear minimum around $B \approx 2.8 \mathrm{~T}$ with a Fermi energy lying between $\mathrm{LL}_{+2}$ and $\mathrm{LL}_{+3}$, while the energetically closest resonant transition $\left(\mathrm{LL}_{+3} \rightarrow \mathrm{LL}_{+4}\right)$ can be found at $B \approx 2.1 \mathrm{~T}$. This shift away from the resonant magnetic field results from cascade pumping of successive inter-Landau-level transitions [see the inset in Fig. 4(b)]. To elucidate this, let us compare the carrier dynamics at both magnetic fields: At the resonance, the situation corresponds to the sketch in Fig. 2(f) (with $n=3$ ). Consequently, the pump pulse off-resonantly excites electrons to $\mathrm{LL}_{n}$ first, thereby enhancing the resonant absorption of the probe pulse, hence the negative DTS. However, if pumping is strong enough, excited electrons in $\mathrm{LL}_{n}$ are further excited to $\mathrm{LL}_{n+1}$. This results in a positive DTS due to absorption bleaching. At higher magnetic fields away from the resonance the LL spacings increase, and the transition $n \rightarrow n+1$ no longer is in resonance with the photons. Instead, the resonance shifts to higher LLs, as shown in the inset of Fig. 4(b). In our example (at $B \approx 2.8 \mathrm{~T}$ ) the transition $n+1 \rightarrow n+2$ is approximately in resonance. Therefore, the second step in the cascade pumping $n \rightarrow n+1$ induces an absorption enhancement leading to a negative DTS. All in all, a shift of the minima away from the resonances indicates that cascade pumping takes place. Its magnitude is a measure of the number of transitions that are involved in the cascade, and it increases with the LL broadening, pump fluence, and magnetic field, i.e., when off-resonant pumping is enhanced. As a side effect of the increased impact of off-resonant pumping at higher $B$, the amplitude of the DTS decreases [see Figs. 4(a) and 4(b)] since positive and negative contributions partly cancel each other.

Note that the pumping cascade moves not only up but also down the LL ladder at the same time since pumping an intraband transition $n \rightarrow n+1$ not only populates $\mathrm{LL}_{n+1}$ but also depopulates $\mathrm{LL}_{n}$, enabling pumping of the transition $n-1 \rightarrow n$ [see the inset in Fig. 4(a)]. As explained above, the upward-moving cascade leads to a shift of the minima to higher magnetic fields for Fermi energies lying below the resonant transition. In close analogy, the downward-moving cascade causes a shift to lower magnetic fields for Fermi energies lying above the resonant transition. This case corresponds to Fig. 2(d), where the transition $n+1 \rightarrow n+2$ is pumped first, before the transition $n \rightarrow n+1$ can be pumped, and so on. Based on the concept of cascade pumping, we can also understand the asymmetry between the two branches in Fig. 4(b): Due to the decreasing energy difference between successive LLs with $n$, the upward-moving cascade [corresponding to the lower branch in Fig. 4(b)] generally evolves faster than the downward-moving cascade (upper branch), which results in a larger shift away from the resonance and in a broader minimum, as is clearly visible in Fig. 4(b).

Since the magnitude of the shift of the minima away from the respective resonant magnetic field depends on the Landaulevel broadening, an estimation of the broadening from the position of the DTS minima is facilitated. Large Landau-level broadenings are beneficial for off-resonant pumping. As a result, cascade pumping is accelerated, increasing the shift of the DTS minima as a function of the magnetic field. Furthermore, our findings demonstrate how to determine the Fermi energy or the pump energy from differential transmission measurements. A recent experiment by Mittendorff et al. [14] showed negative and positive contributions in the differential transmission, which is in line with our calculations. However, the 
measurements are performed on epitaxial graphene samples where a large number of layers with different Fermi energies are present. A direct comparison between experiment and theory suggests that the Fermi energy within the highly doped layers might be spatially inhomogeneous.

\section{CONCLUSION}

In summary, we have presented a theoretical investigation of the intraband carrier dynamics in Landau-quantized graphene excited by low-energy terahertz radiation. Modeling pumpprobe experiments, we have found an interesting dependence of the differential transmission on the Fermi energy and the magnetic field. Our calculations have revealed a positive DTS when the same transition is resonantly pumped and probed. Interestingly, the resonance condition imposes a linear dependence between Fermi energy and magnetic field, where the constant of proportionality is defined by the energy of the excitation field. In contrast, a negative DTS has been found when the resonant transition is strongly Pauli blocked. In this case, different transitions are effectively pumped and probed, resulting in an absorption enhancement and a negative DTS. The gained insights may be utilized in pump-probe experiments to estimate the Landau-level broadening and the Fermi energy of the investigated graphene sample.

\section{ACKNOWLEDGMENTS}

We acknowledge financial support from the Deutsche Forschungsgemeinschaft (DFG) through SPP 1459 and SFB 910. Furthermore, E.M. is grateful to the Swedish Research Council (VR) and the EU Graphene Flagship (CNECT-ICT604391).
[1] M. O. Goerbig, Electronic properties of graphene in a strong magnetic field, Rev. Mod. Phys. 83, 1193 (2011).

[2] M. L. Sadowski, G. Martinez, M. Potemski, C. Berger, and W. A. de Heer, Landau Level Spectroscopy of Ultrathin Graphite Layers, Phys. Rev. Lett. 97, 266405 (2006).

[3] D. S. L. Abergel and V. I. Fal'ko, Optical and magneto-optical far-infrared properties of bilayer graphene, Phys. Rev. B 75, 155430 (2007).

[4] J. Rioux, G. Burkard, and J. E. Sipe, Current injection by coherent one- and two-photon excitation in graphene and its bilayer, Phys. Rev. B 83, 195406 (2011).

[5] P. Plochocka, P. Kossacki, A. Golnik, T. Kazimierczuk, C. Berger, W. A. de Heer, and M. Potemski, Slowing hot-carrier relaxation in graphene using a magnetic field, Phys. Rev. B 80, 245415 (2009).

[6] F. Wendler, A. Knorr, and E. Malic, Resonant carrier-phonon scattering in graphene under Landau quantization, Appl. Phys. Lett. 103, 253117 (2013).

[7] W.-P. Li, J.-W. Yin, Y.-F. Yu, and Z.-W. Wang, Two-phonon relaxation processes of the graphene in the magnetic field, Solid State Commun. 163, 19 (2013).

[8] Z.-W. Wang, L. Liu, L. Shi, X.-J. Gong, W.-P. Li, and K. Xu, The temperature dependence of optical phonon scattering in graphene under strong magnetic field, J. Phys. Soc. Jpn. 82, 094606 (2013).

[9] F. Wendler, A. Knorr, and E. Malic, Carrier multiplication in graphene under Landau quantization, Nat. Commun. 5, 3703 (2014).

[10] F. Wendler and E. Malic, Carrier-phonon scattering in Landauquantized graphene, Phys. Status Solidi B 251, 2541 (2014).

[11] Z.-W. Wang, L. Liu, and Z.-Q. Li, Fast two-phonon relaxation process between the Landau levels of graphene on different polar substrates, Europhys. Lett. 108, 36005 (2014).

[12] F. Wendler and E. Malic, Towards a tunable graphene-based Landau level laser in the terahertz regime, Sci. Rep. 5, 12646 (2015).
[13] M. Mittendorff, F. Wendler, E. Malic, A. Knorr, M. Orlita, M. Potemski, C. Berger, W. A. de Heer, H. Schneider, M. Helm, and S. Winnerl, Carrier dynamics in Landau-quantized graphene featuring strong Auger scattering, Nat. Phys. 11, 75 (2015).

[14] M. Mittendorff, M. Orlita, M. Potemski, C. Berger, W. A. de Heer, H. Schneider, M. Helm, and S. Winnerl, Intraband carrier dynamics in Landau-quantized multilayer epitaxial graphene, New J. Phys. 16, 123021 (2014).

[15] F. Wendler, A. Knorr, and E. Malic, Ultrafast carrier dynamics in Landau-quantized graphene, Nanophotonics 4, 224 (2015).

[16] Y. Wang, M. Tokman, and A. Belyanin, Continuous-wave lasing between Landau levels in graphene, Phys. Rev. A 91, 033821 (2015).

[17] E. Malic and A. Knorr, Graphene and Carbon Nanotubes: Ultrafast Optics and Relaxation Dynamics (Wiley-VCH, Weinheim, Germany, 2013).

[18] E. Malic, T. Winzer, E. Bobkin, and A. Knorr, Microscopic theory of absorption and ultrafast many-particle kinetics in graphene, Phys. Rev. B 84, 205406 (2011).

[19] T. Winzer, A. Knorr, and E. Malic, Carrier multiplication in graphene, Nano Lett. 10, 4839 (2010).

[20] A. H. Castro Neto, F. Guinea, N. M. R. Peres, K. S. Novoselov, and A. K. Geim, The electronic properties of graphene, Rev. Mod. Phys. 81, 109 (2009).

[21] H. Funk, A. Knorr, F. Wendler, and E. Malic, Microscopic view on Landau level broadening mechanisms in graphene, Phys. Rev. B 92, 205428 (2015).

[22] T. Ando and Y. Uemura, Theory of quantum transport in a two-dimensional electron system under magnetic fields. I. Characteristics of level broadening and transport under strong fields, J. Phys. Soc. Jpn. 36, 959 (1974).

[23] F. Wendler, H. Funk, M. Mittendorff, S. Winnerl, M. Helm, A. Knorr, and E. Malic, Efficient Auger scattering in Landauquantized graphene, Proc. SPIE 9361, 936105 (2015). 